\title{
Quantification of N-Terminal Procollagen I Propeptide in Patients With Occlusal Trauma Induced by Prosthetic Factors
}

\author{
ARINA ALICE CIOCAN PENDEFUNDA, COSMIN GABRIEL POPA*, DANIELA ANISTOROAEI, IONUT LUCHIAN, \\ GABRIELA IFTENE, SILVIA MARTU, NORINA CONSUELA FORNA \\ Grigore T. Popa University of Medicine and Pharmacy, Faculty of Dental Medicine, 16 Universitatii Str., lasi, 700115, Romania
}

\begin{abstract}
Occlusal trauma can be generated by a number of local factors, including incorrect dental prosthetic systems. Proper therapy can lead to improved tissue homeostasis, with an increase in bone neoformation, whose markers include the N-terminal procollagen I propeptide (PINP). The purpose of the study was to determine serum N-terminal procollagen I propeptide levels in patients with occlusal trauma caused by incorrect prosthetic systems, performed before and after the establishment of perio-prosthetic complex therapy. The study was performed on a group of 56 patients with periodontal disease and prosthetic systems generating nonphysiological, traumatic occlusal forces. These patients underwent the clinical examination, appropriate multidisciplinary therapy, and N-terminal procollagen I propeptide levels were evaluated in the serum at baseline, 6 months, and 12 months after the therapy completion. The results demonstrated significant improvements for periodontal parameters in both post-treatment assessments and an increase in N-terminal procollagen I propeptide, more significant at one year after treatment.
\end{abstract}

Keywords: N-terminal procollagen I propeptide, occlusal trauma, interdisciplinary treatment

Periodontal disease is a complex form of local disease with possible systemic implications. The theory that periodontal disease is an infectious disease has long been overcome [1,2], and the importance that local and systemic factors have in the etiopathogenicity of periodontal disease being widely renown [3-5].

Among the local factors favouring periodontal disease are also the traumatic occlusal forces. The periodontium tries to adapt to the forces exerted on the crown. This adaptive capacity varies depending on the individual and even in the same person at different times. The effect of occlusal forces on periodontium is influenced by the magnitude, direction, duration and frequency of the forces [6]. When the magnitude of the occlusal forces increases, the periodontium responds with a widening of the periodontal ligament space, an increase in the number and width of the periodontal ligament fibres, and an increase in the alveolar bone density. Changing the direction of the occlusal forces causes a reorientation of the tensions within the periodontium [7]. The main fibres of the periodontal ligament are arranged to fit the occlusal forces best along the long axis of the tooth. Lateral (horizontal) and rotational forces are more prone to periodontal damage. The alveolar bone response is also affected by the duration and frequency of occlusal forces. Constant bone pressure is more damaging than intermittent forces. The more frequent the application of an intermittent force, the more severe is the force for periodontium [8].

Alveolar bone bone destruction can be detected by imaging examinations (radiographs, computed tomography) and by immunohistochemistry exams. The same happens with bone neoformation. There are a number of markers that palpably quantify the level of reconstruction of bone tissue lost through inflammatory pathophysiological phenomena. These markers include the N-terminal procollagen I propeptide.

Occlusal trauma can be generated by a number of local and systemic factors (at CNS level or even a by range of medications). Among the most common local factors are incorrectly made prosthetic devices that induce occlusal interference [9-11]. Trauma from occlusion is reversible.
When trauma is artificially induced in experimental animals, the teeth are removed or intruded into the jaw. When the impact of artificially created force is released, the tissues are repaired.

Although trauma from occlusion is reversible under such conditions, it is not always corrected and is therefore not always temporary or has limited clinical significance [12]. Traumatic force must be removed for the repair to take place. If conditions do not allow teeth to eliminate or adapt to excessive occlusal force, periodontal damage persists and worsens. In addition, the presence of inflammation in periodontitis as a result of plaque accumulation may affect the reversibility of traumatic lesions [13,14].

When the trauma from the occlusion is eliminated, there is a substantial reversal of bone loss, except for the presence of periodontitis. This indicates that inflammation inhibits the bone regeneration potential.

Therefore, it is important to remove the marginal inflammatory component in trauma from occlusion cases because inflammatory phenomena affect bone regeneration after removal of traumatic contacts. It has also been demonstrated in experimental animals that trauma from occlusion does not induce progressive destruction of periodontal tissues in healthy preserved regions after the removal of pre-existing periodontitis [15].

The purpose of the study was to determine the serum levels of N-terminal procollagen I propeptide in patients with occlusal trauma generated by incorrect dental prosthetic systems, performed before and after the establishment of perio-prosthetic complex therapy.

\section{Experimental part}

The study was conducted on a group of 56 patients, at the clinic of Dental Medicine Faculty of Grigore T. Popa UMPh lasi. The inclusion criteria in the study were patients with periodontal disease, patients with fixed prostheses or mobile prostheses generating traumatic occlusal forces. Patients with systemic diseases, smokers, patients who underwent periodontal treatment during the last 12 months, or anti-inflammatory/antibiotic therapy over the last 6 months were excluded from the study. All patients 
were provided with the protocol of the study, under written consent.

Each patient underwent a rigorous and fully dental clinical examination, highlighting the prosthetic therapies in the oral cavity, the quality and correctness of their performance.

The clinical examination included the periodontal examination, measuring the periodontal probing depth, the clinical attachment loss and the degree of bleeding on probing; the clinical examination was complemented by radiological examinations. Following the clinical examination, the complex diagnosis per patient, including the periodontal diagnosis, was also established. All data were recorded in individual patient charts.

To determine the levels of $\mathrm{N}$-terminal procollagen I propeptide (PINP) in the serum, $5 \mathrm{~mL}$ of venous blood were harvested from the antecubital fossa from each patient. Each sample was left for $12 \mathrm{~h}$ at $40^{\circ} \mathrm{C}$ in a separator tube and then centrifuged at $1000 \mathrm{xg}$ for $20 \mathrm{~min}$. Serum samples were placed at $-80^{\circ} \mathrm{C}$ until further processing. The samples were then thawed and processed by ELISA test as instructed by the manufacturer (Cloud-Clone Corp., TX, USA). The detection limit for $\mathrm{N}$-terminal procollagen I propeptide was $33 \mathrm{pg} / \mathrm{mL}$.

After the initial stage of the study, patients underwent complex interdisciplinary therapy, which included nonsurgical periodontal treatment (manual and ultrasonic scaling and root planing), endodontic treatments and prosthetic treatments (repair or even replacement of inappropriate prosthetic systems). Patients were trained in appropriate and individualized oral hygiene methods.

Clinical examination and $\mathrm{N}$-terminal procollagen I propeptide determination in serum were resumed at 6 and 12 months after the completion of therapy.

The data obtained was statistically stored and analysed using IBM SPSS Statistics 25.0. A p value $<0.05$ was considered as statistically significant.

\section{Results and discussions}

The study was conducted on one of 56 patients, 31 of whom were male and 25 females. The initial clinical examination revealed a high percentage of premature contacts in statics $(62.75 \%)$ and also in dynamics (59.33\%). An alarming percentage of $78.57 \%$ of subjects had prosthetic supra-occlusal traumatic design.

From the point of view of the diagnosis of periodontitis, $30.50 \%$ of patients initially presented severe forms of periodontitis, $32.14 \%$ moderate forms and $37.36 \%$ forms of superficial periodontitis (fig.1).
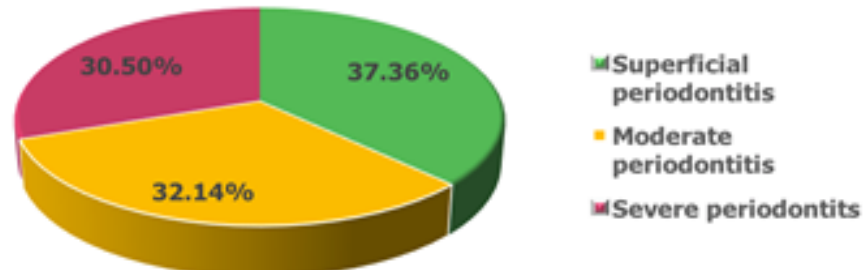

Fig.1 Distribution of periodontitis forms in the study group
Clinical assessments at 6 months after interdisciplinary therapy have shown statistically significant improvements in periodontal parameters, particularly in the periodontal inflammation translated by the bleeding degree on probing.

The serum value of $\mathrm{N}$-terminal procollagen I propeptide, however, did not undergo significant changes, although a slight increase was noted. At 12 months, periodontal parameters followed the clinically detectable improvement pathway, and we also observed a statistically significant increase in $\mathrm{N}$-terminal procollagen I propeptide level versus baseline $(p=0.005)$ (table 1$)$. This fact illustrates at the molecular level, practically, the obvious beneficial effects generated by the complex therapy on the bone reconstruction process.

Periodontitis is an inflammatory disease, mediated by complex interactions between the host and bacterial aggression, which leads to loss of connective tissue attachment, as well as to hard tissue lesions (cement, alveolar bone). After initiation, the disease progresses with loss of collagen fibres and attachment to the surface of the cement, apical migration of the sulcular epithelium, formation of deep periodontal pockets and resorption of the alveolar bone.

The bones are constantly undergoing remodelling processes. In bone remodelling, the bone tissue is constantly resorbed, followed by a bone neoformation phase. In healthy adults, there is a balance between the amount of bone resorbed by osteoclasts and the amount of bone formed by osteoblasts through the coupling mechanism. The coupling process ensures that the destroyed bone volume is equivalent to the bone produced during the subsequent bone formation phase. The concept of bone remodelling is based on the hypothesis that osteoclastic precursors become active and differentiate into osteoclasts, which initiates the bone resorption process. This phase is followed by a bone formation phase [16]. These two stages together determine the turnover rate of the tissue. The number of sites entering the bone formation phase is called the activation frequency.

The need for a periodontal diagnostic tool to provide adequate information for differential diagnosis, disease localization and severity of infection becomes an essential goal in complex patient management. These diagnoses should not only focus on the simple diagnosis of the underlying disease but should also serve as a basis for treatment planning and provide the means to assess the effectiveness of multidisciplinary complex therapy. In affected tissues, biochemical signalling involving three biological phases - inflammation, connective tissue degradation and alveolar bone turnover contributes to clinical morbidity. Circulating molecules in these biological phases have been detected at elevated levels in gingival crevicular fluid, serum, and whole saliva of patients suffering from periodontal disease, making them biomarkers of this disease [17].

Many biomarkers are inflammatory mediators, collagen degradation, and bone turnover related molecules and therefore have emerged as indicators of a possible disease activity. Information on bone remodelling status could be an early indicator of bone destruction leading to progressive

\begin{tabular}{|l|l|l|l|l|l|}
\hline Parameter & Baseline & At 6 months & $\begin{array}{l}p \text { Value (baseline } \\
\text { vs. 6 months) }\end{array}$ & At 12 months & $\begin{array}{l}p \text { Value (baseline vs. } \\
12 \text { months) }\end{array}$ \\
\hline PD (mm) & 7.2 & 6.3 & 0.009 & 4.1 & 0.002 \\
\hline CAL (mm) & 3.8 & 3.1 & 0.02 & 2.3 & 0.003 \\
\hline BOP (\%) seric & $67.25 \%$ & $32.20 \%$ & 0.005 & $20.15 \%$ & 0.001 \\
\hline $\begin{array}{l}\text { PINP } \\
\text { (ng/ml) }\end{array}$ & 32.9 & 0.09 & 38.6 & 0.005 \\
\hline $\begin{array}{l}\text { PD: probing depth; CAL: clinical attachment loss; BOP: bleeding on probing; PINP: N-terminal procollagen I } \\
\text { propeptide }\end{array}$
\end{tabular}

Table 1

CLINICAL PERIODONTAL PARAMETERS AND PINP .EVELS AT BASELINE, AT 6 AND 12 MONTHS POSTTREATMENT 
periodontal disease [18]. Bone remodelling biomarkers may be of two types, bone resorption markers and bone formation markers, reflecting osteoclastic activity and osteoblastic activity, and therefore in most cases any of these markers will reflect a change in the physiological or pathophysiological processes of bone.

A biomarker is a substance used to indicate a biological state. It may be defined as an objectively measured substance evaluated as an indicator of normal biological processes, pathogenic processes or pharmacological responses to a therapeutic intervention.

Non-invasive means of detecting disease activity and treatment outcome may be the most desirable objective in promoting and delivering health causes. Biochemical indicators of bone turnover have proven to be a useful, noninvasive and relatively inexpensive tool for studying bone metabolism in population studies and are gradually established in clinical practice. Their main use, however, is to monitor the response to treatment. Continuous development of new markers of bone turnover will enhance the knowledge of the oral cavity pathophysiology.

Type I collagen is the most abundant collagen in the human body and is predominanty found in bone mineralized tissue. It is a product of two genes, COL1A1 on chromosome 17 and COL1A2 on chromosome 7, which encodes two chains of the collagen molecule, called $\alpha 1$ (I) and $\alpha 2$ (I) chains. Type I collagen deposited in tissues is derived from type I procollagen which is synthesized by fibroblasts and osteoblasts.

Type I procollagen contains at both ends amino terminal and terminal carboxyl propeptides that are cleaved by specific proteases during maturation of collagen. The mature collagen molecule is ready to assemble into collagen fibre, while propeptides are released into the intracellular space and, most of the time, in the circulation. When one mole of collagen is produced, one mole of trimeric propeptides is produced. Specific by-products are synthesized stoichiometrically during type I collagen synthesis and are thus true markers of osteo-synthesis [19].

The molecular weight of the trimeric propeptide is about 35,000 and its constituent chains 14,250 (proal chain) and 5500 ( proa2 chain). The prol chain has two domains, the globular domain resembling the von Willebrand $C$ factor repeat, and a triple helical domain. Proa2 has no globular domain and only has a helical triple domain. In the human serum, N-terminal procollagen I propeptide exists in two forms that can be separated by gel filtration, although their apparentelution positions are much higher than the actual molecular weights. The larger antigen is the trimeric propeptide (PINP intact) and the smaller antigen is the monomeric form, containing only one proa chain [20].

The two PINPs have different catabolic pathways because trimeric $\mathrm{N}$-terminal procollagen I propeptide is rapidly eliminated from blood circulation through scavenger receptors on liver endothelial cells. The clearance of the monomeric form of $\mathrm{N}$-terminal procollagen I propeptide has not been fully studied. However, it is probably eliminated by the kidneys, as demonstrated by the similar monomeric fragment of the collagen type III amino terminal propeptide. From human amniotic fluid, $\mathrm{N}$-terminal procollagen I propeptide can be isolated as foetal antigen 2 and is mainly found in monomeric form [21].

Type I procollagen is synthesized in both bone tissue and soft tissues, and so N-terminal procollagen I propeptide is also produced in these tissues. In bone tissue, $\mathrm{N}$-terminal procollagen I propeptide is released prior to mineralization because there is no $\mathrm{N}$-terminal procollagen I propeptide in the mineralized matrix. However, in soft tissues there may be $\mathrm{pN}$-collagen residues. correlated with N-terminal procollagen I propeptide, which proved to be a sensitive marker of bone formation. Radiographic alveolar bone changes were observed in 57\% of the participants in a study by Jagur et al. [22].

This suggests that low levels of N-terminal procollagen I propeptide interfere with maxillary bone formation, causing erosions, lesions and osteophytes. In our study, complex, prosthetic, periodontal and endodontic therapy generated elevations in $\mathrm{N}$-terminal procollagen I propeptide serum levels, more evident 12 months after therapy.

Of course, additional, comparative and prospective studies are needed on wider study groups and a greater diversity of molecules that can essentially function as markers of complete and complex therapeutic success.

\section{Conclusions}

Multidisciplinary therapy in patients with periodontitis and occlusal trauma generated by dental prosthetic factors caused significant improvements in both clinical and serum $\mathrm{N}$-terminal procollagen I propeptide, providing the molecular image of the initiation of the hard tissue neoformation process.

\section{References}

1.MARTU, I., GORIUC, A., MARTU, M.A., VATA, I., BACIU, R., MOCANU, R., SURDU, A.E., POPA, C., LUCHIAN, I., Rev Chim (Bucharest), 68, no.10, 2017, p.2407-12

2.MARTU, M.A., SOLOMON, S.M., SUFARU, I.G., JELIHOVSCHI, I., MARTU, S., REZUS, E., SURDU, A.E., ONEA, R.M., GRECU, G.P., FOIA, L., Rev. Chim. (Bucharest), 68, no.8, 2017, p.1946-1949

3.NICOLAICIUC, O., et al. Rev. Chim. (Bucharest), 68, no.3, 2017, p.619-623.

4.VEISA, G., et al. Rev. Chim. (Bucharest), 67, no.1, 2016, p.103-105. 5.SOLOMON S. et al. Int. J. Clin. Exp. Med., 2016, 9(2), pp.4411-4417. 6.LUCHIAN I, VATA I, MARTU I, TATARCIUC M, PENDEFUNDA V, MARTU S. Rom J of Oral Rehab, 2016; 8(1):80-83.

7.NEW MANN M., TAKEI H., KLOKKEVOLD P., CARRANZA F. Carranza's Clinical Periodontology 12th Edition Saunders 2015.

8.SOLOMON S.M., IOVAN G., PASARIN L., SUFARU G.I., MARTU I., LUCHIAN I., MARTU M.A., MARTU S. Rom. J. Oral Rehab., 2017, 9(3): 89-96.

9.ZEGAN G, DASCALU CG, MAVRU RB.; etal. Medical-Surgical J ournalRevista Medico-Chirurgicala, 2015, vol. 119, Issue: 4: 1153-1160

10.ANISTOROAIE, D., MATEI, M.N., PACURAR, M., et al. Rev. Chim.(Bucharest), 69, no. 11, 2018, p. 4210-4214

11.MARTU I, LUCHIAN I, DIACONU-POPA D, DOSCAS AR, BOSANCEANU DG, VITALARIU A, LUCA 0, TATARCIUC M. Rom. J. Oral Rehab.,2017, 9(1):27-31.

12.POPA C et al. Int. J. Med. Dent., 2018, 8, pp.83-88.

13.SOLOMON, S.M., STOLERIU, S., AGOP FORNA ,D., et al , Mat. Plast., 53, no.2, 2016, p. 304-307

14.LIU H, JIANG H, WANG H. J. Oral. Rehab., 2013, 40, pp.130-138. 15.FAN J, CATON J G. J. Clin. Periodontol., 2018, 45(Suppl 20), pp.S207S218.

16.SINCAR DC et al. Rom. J. Oral. Rehab., 2018, 10, pp.26-31

17.RAM VS et al. J. Clin. Diagn. Res., 2015, 9(1), pp.ZE07-ZE10.

18.LUCHIAN I et al. Rom. J. Oral. Rehab., 2018, 10(4), pp.60-70. 19.RISTELI J, RISTELI L. In: SEIBEL MJ, ROBINS SP, BILIZIKIAN RJP, eds. Dynamics of Bone and Cartilage Metabolism: Principles and Clinical Applications. 2nd edn. Chapter 23. Academic Press: San Diego, CA, USA, 2006

20.KOIVULA MK et al. Ann. Clin. Biochem., 2010, 47, pp.67-71. 21.GARNERO P, VERGNAUD P, HOYLE N. Clin. Chem., 2008, 54, pp.18896.

22. J AGUR 0 et al. Baltic. Dent. Maxillofac. J., 2011, 13, pp.42-48.

\section{Manuscript received: 18.08 .2018}

